\section{Patterns of, and factors associated with, atypical and typical antipsychotic prescribing by general practitioners in the UK during the 1990s}

PSSRU

R E S E A R C H $S$ U M M A R Y

\section{BACKGROUND}

The development of atypical (or second generation) antipsychotics has offered people with schizophrenia more treatment options. Atypical antipsychotics are judged to be more effective in alleviating symptoms of the illness and to be associated with fewer side-effects in most patients. They are more expensive than the earlier class of antipsychotics, so-called typical antipsychotics, but doubt remains as to whether or not they are more cost-effective in treating patients with schizophrenia.

Spending on antipsychotics in the United Kingdom was ten times greater in 2002 than it was in 1996, a trend matched in much of Europe. The introduction and uptake of atypical antispychotics has been the primary reason. And in July 2002, the National Institute for Clinical Excellence (NICE) published guidance favouring the use of atypical antipsychotics as a first-line treatment for patients with schizophrenia, which has contributed to an increase in the rate of prescribing of atypicals relative to typicals and increased the proportion of mental health drug spending that goes towards prescribing antipsychotics.

\section{THE STUDY}

Using the General Practice Research Database (GPRD) we assess changes in the prescribing of atypical antipsychotics in the treatment of schizophrenia and schizoaffective disorder over time and explore associations between individual characteristics and the changes in prescribing patterns.

The GPRD is a computerised database of anonymised general practice patient records. It began in 1987 and now contains over 30 million patient years of information. The database currently collects information on approximately 3 million patients - approximately $4.7 \%$ of the UK population - provided by a cross-section of practices, drawn from across the UK.

To assess the prescribing patterns of atypical antipsychotics in the early part of their availability, the study used data from January 1993 through to December 1999. Atypical antipsychotic medications were not prescribed in the UK before 1993 and problems with the dataset that occurred in 2000 and 2001 resulted in a significantly reduced pool of observations. Our analysis linked annual GPRD datasets from one year to the next for individual patients based on their unique patient identification number.

Our sample includes every individual who received at least one prescription for an antipsychotic medication, who was enrolled in the General Practice Research Database for the entire year and had been diagnosed with schizophrenia or schizoaffective disorder prior to the beginning of that year. There were no other inclusion or exclusion criteria. The individual patient characteristics considered in our model of the prescribing choice over time were: age, sex, number of years since schizophrenia or schizoaffective disorder diagnosis, whether or not they had been an inpatient in the previous year and number of GP visits in the previous year.

\section{RESULTS}

A total of 4,391 patients contributed data to our analysis. Of the patients who contributed data in 1993, $1.8 \%$ were prescribed an atypical antipsychotic, but by 1999 , the rate had increased to $20.8 \%$ (see Figure 1). This rate of growth is comparable to UK data from other sources, although none appear to have looked at as wide a sample as we were able to examine.

Data from the NHS Prescription Pricing Authority indicates that the rate of atypical antipsychotic prescribing in General Practice in England has increased substantially since the period observed in our study, from $21.3 \%$ in June 2000 to $61.1 \%$ in June 2005. These data are not strictly comparable to the GPRD data, however, as they include prescribing for indications other than schizophrenia and schizoaffective disorder and relate to rates in England only, as compared to the GPRD which incorporates data for all of the UK.
Our analysis identified three significant correlates of prescription choice: age, previous inpatient stay and previous frequency of primary care consultation. Older patients were less likely to be prescribed an atypical as compared to a typical antipsychotic. And those who in the last year had either an inpatient stay or a high frequency of GP visits were more likely to be prescribed an atypical as compared to a typical antipsychotic. Gender and time since first diagnosis of schizophrenia or schizoaffective disorder were not associated with higher or lower odds of being prescribed an atypical antipsychotic.

\section{Age}

A five-year increase in age was associated with a decrease by $15 \%$ in the probability of being prescribed an atypical antipsychotic. So, for example, all other things being equal, a 35 year old would be $15 \%$ less likely to be prescribed an atypical antipsychotic as compared to a 30 year old. Possible explanations for this effect are that (i) younger patients in the sample, if newly diagnosed, are more likely be prescribed an atypical antipsychotics as their initial prescription; (ii) GPs may be more defensive in their prescribing to older patients, due to initial warnings that atypical antipsychotics increase the risk of ischaemic stroke in older patients (recent research has concluded no

\section{Figure 1 Per cent of patients prescribed atypical antipsychotics}

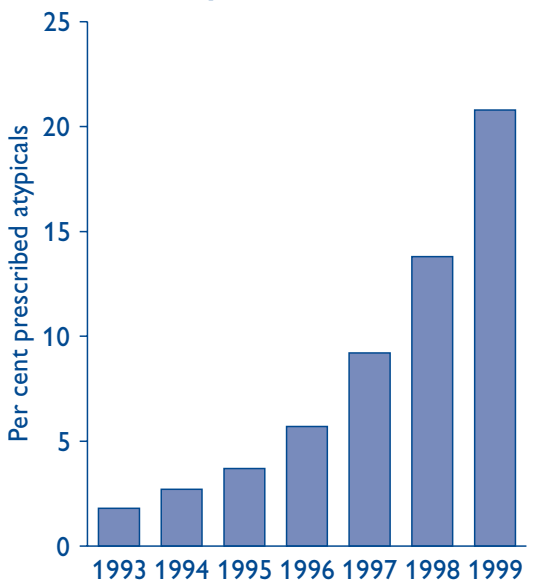




\section{Box 1. Main findings}

- In the General Practice Research Database, atypical antipsychotics as a percentage of antipsychotic prescribing by GPs for schizophrenia and schizoaffective disorder increased from $1.8 \%$ in 1993 to $20.8 \%$ in 1999 .

- Older schizophrenia and schizoaffective disorder patients were significantly less likely to be prescribed an atypical antipsychotic relative to a typical antipsychotic.

- Patients who had an inpatient stay in the previous year were significantly more likely to be prescribed an atypical antipsychotic.

- Patients who had a high number of GP visits in the previous year were significantly more likely to be prescribed an atypical antipsychotic.

- The prescribing choice made by GPs in treating patients with schizophrenia or schizoaffective disorder was not made solely on needs-based criteria.

significant difference exists in the risk of ischaemic stroke for dementia patients taking atypical antipsychotics as compared to typical antipsychotics); or (iii) older patients, having had their illness for a longer period of time (the age of onset of schizophrenia being almost always in early adulthood), were more likely to have been stabilised on a typical antipsychotic and therefore less likely to be prescribed an atypical antipsychotic (however, a test of the effect of time since diagnosis found this effect not statistically significant).

\section{Inpatient stays in the previous 12 months}

Patients who had an inpatient stay in the previous year were over one and a half times as likely to receive atypical antipsychotics as compared to a typical antipsychotic. If it is reasonable to assume that inpatient admission follows an exacerbation of symptoms, then it can be inferred that patients with more severe illness, or whose illness has not been stabilised, are more likely to be prescribed atypical antipsychotics. Alternatively, this result may reflect a preference of prescribing atypical antipsychotics, for acute patients, by hospital-based psychiatrists. The prescription choice would then be continued by the patient's GP once they were discharged from hospital.

\section{GP visits in the previous 12 months}

Patients who had visited their GP six or more times in the previous year were over one and a half times as likely to receive atypical antipsychotics as compared to a typical antipsychotic. This may reflect that patients presenting with more complex needs were more likely to be prescribed atypicals. Another possibility is that a high number of GP visits reflect problems with side-effects that may induce a switch to an atypical antipsychotic.

The setting of care may also be a key factor in the prescription choice. Just as hospital psychiatrists may prescribe more atypical antipsychotics as they are often treating acutely ill patients, GPs may prefer using the older typical antipsychotics as they are more likely to be familiar with these drugs. As the GPRD data are primary care-based, there may be a bias in lower rates of atypical antipsychotic prescribing in these data.

\section{SUMMARY}

The trend of an increase in the rate of atypical antipsychotic prescribing in schizophrenia, as a percentage of all antipsychotic prescribing, is likely to continue. Reductions in their real prices, relative to typical antipsychotics, seems likely to encourage GPs to prescribe atypical antipsychotics more frequently in preference to the older medications. The modelling results indicate that in prescribing for patients with schizophrenia or schizoaffective disorder, it is the case that factors not directly indicative of need were being used by GPs in making the choice between atypical and typical antipsychotics.

This study is potentially limited by the scope and content of the source of data.
The GPRD includes prescribing data on prescribing initiated by the GP or by a specialist in an outpatient setting. The dataset does not include records of hospital inpatient prescribing, which may represent a sizeable proportion of antipsychotic prescribing. Data from IMS Health on sales of atypical antipsychotic in the United Kingdom indicate that in 2002 approximately $33 \%$ of prescriptions for atypical antipsychotics take place within hospitals.

Ideally, we would also have included more detailed data on the characteristics of patients, and medical practitioners, but these data were not available. It has been suggested by other UK studies that receipt of an atypical rather than a typical antipsychotic is linked to factors such as treatment history, risk of non-adherence and ethnicity.

The impact of the NICE guidance on the rate of increase in atypical antipsychotic prescribing and on the transparency in the prescribing choice are interesting issues for further research. Additional analyses are also necessary to determine whether or not these trends are consistent across the atypical class of antipsychotics.

\section{Acknowledgements}

This work was funded by the Department of Health as part of the PSSRU's programme of mental health research.

\section{Further information}

See King, D. and Knapp, M. (2006) Patterns of, and factors associated with, atypical and typical antipsychotic prescribing by general practitioners in the UK during the 1990 s, Journal of Mental Health, 15, 269-278.

\section{Authors}

Derek King (PSSRU, LSE) and Martin Knapp (PSSRU, LSE and Centre for the Economics of Mental Health, Institute of Psychiatry, King's College London).

The PERSONAL SOCIAL SERVICES RESEARCH UNIT undertakes social and health care research, supported mainly by the Department of Health, and focusing particularly on policy research and analysis of equity and efficiency in community care, long-term care and related areas - including services for elderly people, people with mental health problems and children in care. Views expressed in PSSRU publications do not necessarily reflect those of funding organisations. The PSSRU was established at the University of Kent at Canterbury in 1974, and from 1996 it has operated from three branches:

Cornwallis Building, University of Kent, Canterbury, Kent, CT2 7NF

London School of Economics and Political Science, Houghton Street, London, WC2A 2AE

University of Manchester, Dover Street Building, Oxford Road, Manchester, M13 9PL

The PSSRU Bulletin and publication lists are available free from the unit librarian in Canterbury (01227 827773; e-mail pssru_library@kent.ac.uk) and on the PSSRU website. 